UniversitätSpital Zürich

Klinik für Mund-, Kiefer- und Gesichtschirurgie

Direktor: Prof. Dr. med. Dr. med. dent. Martin Rücker

Arbeit unter Leitung von Dr. med. Dr. med. dent. Thomas Gander

\title{
Inferior alveolar nerve function after open reduction and internal fixation of mandibular fractures
}

\author{
INAUGURAL- DISSERTATION \\ zur Erlangung der Doktorwürde der Zahnmedizin \\ der Medizinischen Fakultät \\ der Universität Zürich
}

vorgelegt von

Jan Samuel Schenkel

Genehmigt auf Antrag von Prof. Dr. med. Dr. med. dent. Martin Rücker

Zürich 2016 


\section{Publikationshinweis}

Inferior alveolar nerve function after open reduction and internal fixation of mandibular fractures

Publiziert am: Juni 2016

Journal: Journal of Cranio-Maxillo-Facial Surgery, Volume 44, Issue 4, Pages 743-748 


\title{
Inferior alveolar nerve function after open reduction and internal fixation of mandibular fractures
}

\author{
Jan Samuel Schenkel, Christine Jacobsen, Claudio Rostetter, Klaus W. Grätz, Martin Rücker, \\ Thomas Gander*
}

Department of Cranio-Maxillofacial and Oral Surgery, University Hospital of Zurich, Frauenklinikstrasse 24, CH-8091 Zurich, Switzerland

\section{A R T I C L E I N F O}

\section{Article history:}

Paper received 15 September 2015

Accepted 11 March 2016

Available online 19 March 2016

\section{Keywords:}

Inferior alveolar nerve injury

Hypoesthesia

Mandibular fractures

Open reduction

Fixation

\begin{abstract}
A B S T R A C T
Purpose: Mandibular fractures are amongst the most common facial fractures and are usually treated by open reduction and internal fixation (ORIF). Inferior alveolar nerve (IAN) injuries are seen frequently in mandibular fractures as well as after ORIF of these fractures due to the exposition and the close proximity of the nerve during fracture reduction. Therefore the continuity of the IAN can be disrupted. Permanent injury to the IAN can result in diminished quality of life. This retrospective study was designed to objectively analyse the incidence and the outcome of pre- and postoperative mental nerve hypoesthesia after ORIF of mandibular fractures.

Material and methods: Patients who were consecutively treated at the Department of Cranio-Maxillofacial and Oral Surgery of the University Hospital Zurich between 2004 and 2010 with mandibular fractures who underwent ORIF were included. Follow-up period was 12 months. Demographic, pre-, peri- and postsurgical data were tabulated and statistically evaluated using the $\chi^{2}$ test and the Kruskall-Wallis-Test.

Results: 340 patients met the inclusion criteria. $27 \%$ of the study population presented with postinjury (preoperative) mental nerve hypoesthesia, $46 \%$ suffered from purely postoperative hypoesthesia and $27 \%$ showed no nerve damage. Complete recovery was seen in $70 \%$ of all cases, partial recovery in $20 \%$ of the cases and less than $10 \%$ suffered from a permanent ( $>12$ months) IAN damage. Mandibular angle fractures were accompanied with significantly higher rates of hypoesthesia (79\% vs. 68\%). Recovery rate was significantly worse in older patients, when preoperative hypoesthesia was present (66\% vs. $73 \%$ ) and in patients with multiple fractures in proximity to the IAN (36\% vs. 52\%). Mandibular body fractures showed worse recovery rates than fractures that did not affect the body (44\% vs. 52\%).

Conclusion: The present study shows that IAN injury is seen frequently in mandibular fractures. Mental nerve hypoesthesia may influence quality of life. Nerve continuity may not be preserved due to the initial trauma or may result as a postoperative complication. Nevertheless the results of this study show a high potential for full recovery.
\end{abstract}

(c) 2016 European Association for Cranio-Maxillo-Facial Surgery. Published by Elsevier Ltd. All rights reserved.

\section{Introduction}

Mandibular fractures are among the most common facial injuries (Boffano et al., 2015). As literature shows, aetiology is country as well as age dependent, but major causes include motor vehicle crashes, work-related injuries, assaults, or sports-related injuries (Gassner et al., 2004; Erdmann et al., 2008; Iatrou et al., 2010). Treatment options include ORIF (open reduction and internal fixation) by either the intraoral or the extraoral approach.

\footnotetext{
* Corresponding author. Tel./fax: +41442550062.

E-mail address: thomas.gander@usz.ch (T. Gander).
}

Conservative treatment, consisting of closed reduction and mandibulo-maxillary fixation (MMF), may be indicated depending on the fracture site and the degree of fragmentation. Modern treatment principles for mandibular fractures in the symphyseal, the body, the angle and the ramus region evidently tend toward rigid internal fixation as fracture fragments can be reduced to reestablish the pretraumatic facial profile (Alpert et al., 2009; Chrcanovic, 2013).

Common complications after ORIF of mandibular fractures include nerve trauma, disturbed wound-healing, infection, malocclusion and non-union (Renton and Wiesenfeld, 1996; Seemann et al., 2010). One of the most common postoperative complications after ORIF of mandibular fractures is mental nerve 
hypoesthesia, which can be of transient or permanent nature. The inferior alveolar nerve (IAN) is at high risk in these fractures due to the exposition to bony fragments, which can cause compression, straining or tearing of the nerve. Therefore the continuity of the nerve may be partially or fully injured.

Risk factors for posttraumatic IAN dysfunction are highly displaced fractures, such as comminuted fractures, and fractures with close proximity to the inferior alveolar nerve (angle, ramus and body) (Bede et al., 2012; Boffano et al., 2014). Literature shows postinjury (preoperative) hypoesthesia rates of up to $81 \%$ (Halpern et al., 2004; Bede et al., 2012). When preoperative alveolar nerve function is intact, the literature demonstrates postoperative hypoesthesia rates around 30\% (Schultze-Mosgau et al., 1999; Renzi et al., 2004) caused by intraoperative irritation during ORIF. Recovery rates of the inferior alveolar nerve between 33\% (Marchena et al., 1998) and 100\% (Mayrink et al., 2013) have been published, which suggests a high potential for recovery. Nevertheless disrupted continuity of the inferior alveolar nerve may cause trouble with chewing, eating, swallowing, smiling, or drooling (Lemke et al., 1998) due to diminished or complete loss of sensitivity of the skin and the mucous membranes.

IAN injury can result in severe reduction of quality of life and in chronic pain (Smith et al., 2013). In a study performed by Marchena et al., 1998, 55\% of patients with persistent sensory deficit felt disturbed by the absence of nerve recovery.

It is therefore important to analyse the preoperative as well as the postoperative IAN function and to inform patients about possible recovery rates. Different methods of assessing the IAN function have been suggested in the literature, such as the sharp/ blunt discrimination, two point discrimination, temperature testing, and others (Poort et al., 2009). While the more elaborate tests are more accurate it can be difficult to incorporate them into a quick postoperative follow-up, which might explain why simple tests are used more routinely.

Nerve lesions can be categorized as neurapraxia, axonotmesis and neurotmesis according to the Seddon classification. Neurapraxia is the mildest nerve injury with intact axon anatomy and shows spontaneous recovery within a few weeks. In axonotmesis the axon is damaged to some degree but recovery without surgical intervention is still possible. In neurotmesis the nerve is completely divided and no spontaneous recovery is possible (Seddon, 1942; Chhabra et al., 2014). The Sunderland classification groups nerve injury into five different groups. A Sunderland first degree injury corresponds to neurapraxia. A Sunderland second degree damage describes axonal damage with full recovery. A Sunderland third degree injury usually recovers in months but surgical intervention may be needed. In Sunderland fourth degree injury recovery only occurs if surgery is performed. Sunderland fifth degree nerve damage corresponds to neurotmesis (Sunderland, 1951). These classifications can help to forecast a prognosis for nerve recovery.

This retrospective study was designed to objectively analyse the incidence and the outcome of mental nerve hypoesthesia after mandibular fractures and after ORIF of these injuries. It was hypothesized that mental nerve hypoesthesia is a common condition after mandibular fractures and that its recovery potential is generally high without special treatment executed.

\section{Material and methods}

This retrospective analysis was performed in concordance with the Swiss Ethical committee (ref.: \# 2014-0341). Patients who were consecutively treated at the Department of Cranio-Maxillofacial and Oral Surgery between 2004 and 2010 with mandibular fractures who underwent ORIF by an intraoral approach were included. Inclusion criteria were defined as:
1) Patient presented between the years 2004 through 2010 with a mandibular fracture at the University Hospital Zurich

2) Only patients with at least one fracture site where the continuity of the IAN might have been injured were included (ramus, angle, body, parasymphyseal and symphyseal region). The anatomical regions for this study were defined as:

- Symphyseal region: area between the lateral incisors

- Parasymphyseal region: area between the lateral incisor and the second premolar

- Body: between the second premolar and the third molar

- Angle: distal of the third molar

- Ramus: area between the mandibular angle and the mandibular notch

3) Treatment was performed by ORIF with an intraoral approach and in fractures of the angle and the ramus a transbuccal incision supported the intraoral approach.

4) Sufficient radiological and clinical documentation with 12 months of follow-up was available

5) IAN function was routinely assessed by sharp/blunt and two point discrimination of the mental nerve. However hypoesthesia of the mental nerve does not automatically mean that there is hypoesthesia of the IAN.

All patients were treated by the AO/ASIF (Arbeitsgemeinschaft für Osteosynthesefragen/Association for the Study of Internal Fixation) guidelines by an intraoral approach. A transbuccal incision to support the intraoral approach has been used for angle and ramus fractures. Fractures were anatomically reduced and internal fixation was performed with the Synthes Mandible or the Medartis osteosynthesis system. In general two mini-plates were used for each fracture, one at the inferior border of the mandible with bicortical screws and one at the superior crestal part with monocortical screws. Non-dislocated fractures of the mandibular angle were treated with a Champy plate on the oblique ridge. At least two screws on each side of the fracture gap were placed.

Demographic information was tabulated including the patients' age, gender and diagnosis. Fracture site, pre- and postoperative mental nerve function as well as period to recovery were extracted from the hospital information system and coded in Excel (Microsoft Excel, Microsoft Corp., Redmond, Washington, USA). The postoperative follow-up interval was 12 months.

Data were then analysed with the Statistical Package for the Social Sciences software (IBM Corp. SPSS Statistics Version 21.0, Chicago, Illinois, USA). Descriptive statistics such as mean, standard deviations, medians, IQRs as well as relative frequencies were computed. Associations between two discrete variables were investigated by means of a Chi-square test. Differences in medians between four groups with respect to continuous variables were analysed by means of a KruskallWallis-Test. Results of statistical analysis with p-values smaller than 0.05 were considered statistically significant.

\section{Results}

\subsection{Demographics}

In total 340 patients met the study's inclusion criteria and could be evaluated. The majority of the patients (79\%, $n=268$ ) were of male gender. Patients were between 18 and 90 years old, with a mean and median age of 33 and 27 years, respectively and with an interquartile range of 21 years.

\subsection{Fractures and nerve injuries}

While every patient included into this study suffered from at least one fracture in close proximity to the IAN (defined in the inclusion 
criteria as: ramus, angle, body, parasymphyseal and symphyseal region), the most common fractures were parasymphyseal $(59 \%$ $\mathrm{n}=199)$, angle $(48 \% \mathrm{n}=163)$ and unilateral collum fractures $(27 \%$ $\mathrm{n}=91)$. Other involved sites were the symphyseal region $(8 \% \mathrm{n}=27)$, the body $(7 \% \mathrm{n}=25)$ and bilateral condylar process fractures $(7 \%$ $\mathrm{n}=22)$. Rare fracture sites in this study were the condylar head ( $2 \%$ $n=6)$, the ramus $(2 \% n=6)$, bilateral body fractures $(1 \% n=3)$ and the coronoid process, the angle bilaterally and the parasymphyseal region bilaterally (each $<1 \% \mathrm{n}=2$ ).

Hypoesthesia of the inferior alveolar nerve was a common finding as shown in Fig. 1. Over one quarter of the patients $(27 \%$, $\mathrm{n}=91$ ) presented with postinjury (preoperative) hypoesthesia, $46 \%$ of the patients ( $n=158$ ) suffered from purely postoperative hypoesthesia, meaning the hypoesthesia was caused intraoperative due to ORIF. In $27 \%(n=91)$ the nerve function was completely intact preoperative and postoperative. All patients with preoperative hypoesthesia had postoperative hypoesthesia as well. If the patients with postinjury (preoperative) and the patients with purely postoperative hypoesthesia are combined, $73 \%(n=249)$ of the study population showed hypoesthesia of the mental nerve. No cases of IAN anaesthesia or completely disrupted nerve continuity were found in this study. The left and the right IAN were involved to a similar extent ( $44 \%$ on the left $n=110$ versus $39 \%$ on the right $n=96)$. Both sides of the IAN were involved in $17 \%(n=43)$.

Analysis of the fracture aetiology was performed for all three groups (hypoesthesia preoperative, hypoesthesia postoperative, no hypoesthesia). The most common cause for mandibular fractures in this study population were interpersonal altercations (39\%, $\mathrm{n}=131)$. The second and third most common reasons were falls $(18 \%, \mathrm{n}=62)$ and traffic accidents $(18 \%, \mathrm{n}=61$, bicycle accidents included). Over $50 \%(n=47)$ of the patients suffering from preoperative (posttraumatic) hypoesthesia had a mandibular fracture due to an interpersonal altercation. Nonetheless, no statistical significance was detected between the groups with preoperative and postoperative hypoesthesia and the fracture aetiology ( $p$ value: 0.060). Furthermore, the patients with neither preoperative nor postoperative hypoesthesia did not show a significantly different fracture mechanism. The most common aetiology for this group were interpersonal altercations ( $30 \%, \mathrm{n}=27$ ), followed by traffic accidents $(23 \%, \mathrm{n}=21)$ and falls $(22 \%, \mathrm{n}=20)$.

\subsection{Postoperative course and follow-up}

Complete recovery of the IAN was seen in the majority of the cases $(70 \% n=175)$ and partial recovery was gained in $20 \%(n=51)$. Less than $10 \%(n=23)$ presented with permanently disturbed IAN nerve function assessed by sharp/blunt and two point discrimination within the follow-up period of 12 months. An overview can be found in Fig. 2. The period to complete or partial recovery was less than 6 months in almost half of the patients suffering from hypoesthesia $(45 \% n=113)$ while in another $45 \%(n=113)$ it was between 6 and 12 months. The remaining 23 patients $(<10 \%)$ with hypoesthesia did not show any recovery in the follow-up period of 12 months. No interventions such as a nerve reconstruction were performed in the group with only partial or no recovery, since there were only cases with hypoesthesia and no patient suffered of complete anaesthesia.

\subsection{Influencing factors for hypoesthesia}

Patients who presented with a fracture in the mandibular angle were found to have a significantly ( $p$-value $<0.001$ ) higher rate of hypoesthesia than patients without angle fractures. Those with fractured angles complained of hypoesthesia in $79 \%(n=128,62$ pre- and 66 postoperatively) while patients without fractures in the angle region presented with hypoesthesia in $68 \%(n=121,29$ preand 92 postoperatively). No statistical significance could be detected between hypoesthesia and multiple fractures in proximity to the IAN. Neither the gender ( $p$-value: 0.8 ) nor the age ( $p$-value: 0.1 ) had a statistical influence on the rate of hypoesthesia.

\subsection{Influencing factors for recovery rate}

The recovery rate was significantly (p-value $<0.001$ ) influenced by the patients' age. Younger patients had a greater potential for recovery than did older patients. Those who did not suffer from IAN injury had a mean age of 30 years (median 24 years) while patients

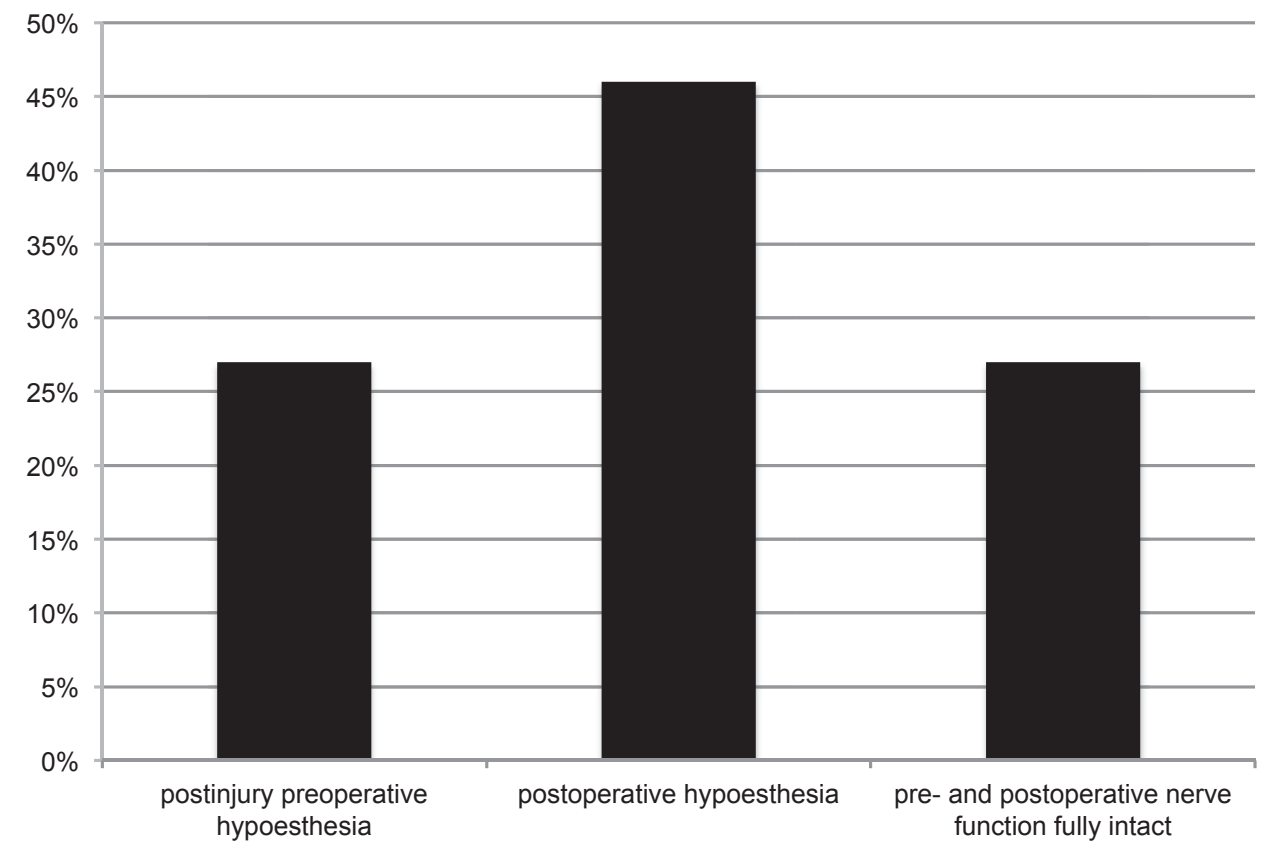

Fig. 1. Shown are the rates of preoperative and postoperative hypoesthesia of the mental nerve as well as the rate of intact inferior alveolar nerve function. 


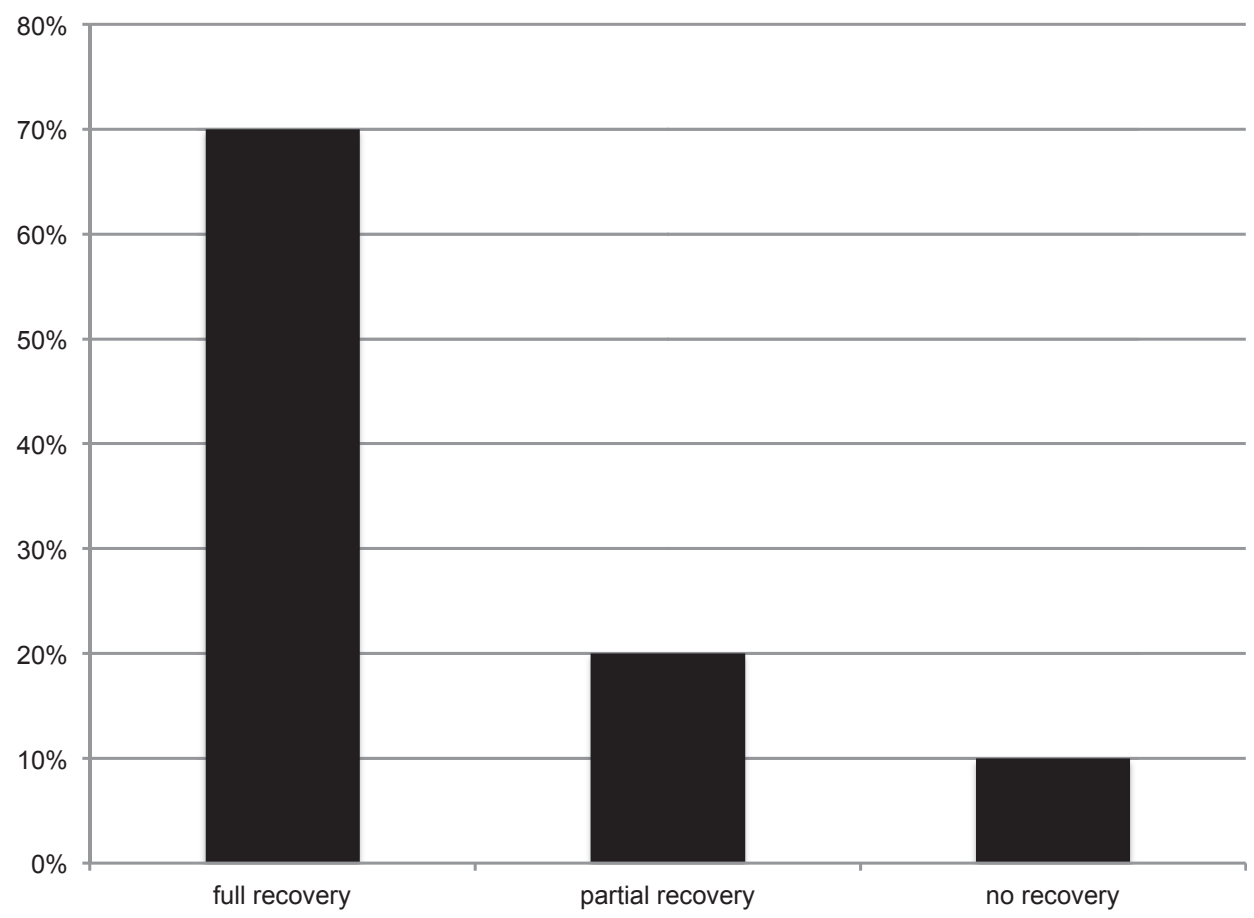

Fig. 2. Illustrated are the recovery rates after inferior alveolar nerve injury with a follow-up period of 12 months.

with complete recovery averaged 31 years (median 25 years). The mean age for patients with only partial IAN recovery and those without recovery was 40 years (with a median of 38 and 36 years respectively).

Furthermore there was a significant difference ( $p$-value $<0.001$ ) in the recovery rate if hypoesthesia was present pre- or postoperative as presented in Fig. 3. Two thirds $(66 \% n=60)$ of the patients with existent preoperative hypoesthesia showed complete recovery while almost three-fourths of the patients with only postoperative hypoesthesia ( $73 \% \mathrm{n}=115)$ developed full recovery. Those with preoperative hypoesthesia were more prone to develop only partial recovery $(22 \% \mathrm{n}=20$ vs. $20 \% \mathrm{n}=31)$ or no recovery at all $(12 \% n=11$ vs. $8 \% n=12)$ when compared to those with postoperative hypoesthesia. The recovery rate was significantly worse ( $p$-value 0.035 ) when patients had more than one fracture in proximity to the IAN (ramus, angle, body, parasymphyseal and symphyseal region). In the group with multiple fractures complete recovery of the IAN was seen in just $36 \%(n=4)$ while over the half of the patients with only one fracture in proximity to the IAN showed full recovery $(52 \% \mathrm{n}=171)$.

The only fracture site that showed significantly ( $p$-value 0.032 ) worse recovery rates was the body of the mandible. In these patients full recovery was seen in less than half of the cases (44\% $\mathrm{n}=11$ versus $52 \% \mathrm{n}=164$ in patients without fractures in the body area). $32 \%(n=8)$ of patients with body fractures showed partial recovery versus $14 \%(n=43)$ in patients without fractures in this site. No recovery was observed in $12 \%(n=3)$ of the group with body fractures while only $6 \%(n=20)$ of the patients without body fractures showed no recovery. No IAN lesions were seen in only $12 \%$ $(n=3)$ in the mandibular body group versus $28 \%(n=88)$ in the group without fractures in the body area. On the other hand the recovery rate was not influenced by the gender (p-value: 0.100 ).

\section{Discussion}

As shown in this study and in the literature, disruption of the IAN continuity is a common complication after mandibular fractures as well as after its operative treatment. Almost one third of the study population showed postinjury preoperative mental nerve hypoesthesia and over 45\% suffered from purely postoperative hypoesthesia without pre-existing posttraumatic nerve injury. This finding correlates with other studies that analysed the IAN function after mandibular fractures (Schultze-Mosgau et al., 1999; Halpern et al., 2004; Renzi et al., 2004; Bede et al., 2012).

Fortunately the literature shows a high potential for recovery after disturbed IAN continuity with published recovery rates between 33\% (Marchena et al., 1998) and 100\% (Mayrink et al., 2013). Analysis of the results of this publication yielded full recovery in $70 \%$ and partial recovery in $20 \%$, leaving only $10 \%$ with no improvement within 12 months after disruption of the IAN continuity. This number might be even lower if the follow-up period of 12 months was extended, since neural regeneration is slow. The large diversity in the published recovery rates might be explained due to the different methods of assessing the IAN function, as suggested by Poort et al., 2009. Sharp/blunt and two point discrimination as well as patient reporting was the method routinely used to check for IAN function in this study, which is one of the publication's limitations.

None of the patients in this study received any treatment if hypoesthesia persisted for over 12 months. No case of complete anaesthesia or complete nerve transection has been observed in this study population, which explains the avoidance of nerve reconstruction techniques.

The analysis of the data revealed that almost one third of the study population did not show any signs of hypoesthesia during the whole treatment course. It is of special interest to know if this group has a different fracture epidemiology than patients with hypoesthesia. However, this study could not reveal any significant differences between the different groups regarding age, fracture aetiology or gender. However, literature shows several risk factors for IAN damage. Two studies, one performed by Bede et al., 2012, the other by Boffano et al., 2014, analysed that highly displaced fractures, such as comminuted fractures and fractures in close proximity to the inferior alveolar nerve (angle, ramus and body) are associated with higher posttraumatic inferior alveolar nerve injury rates. The 


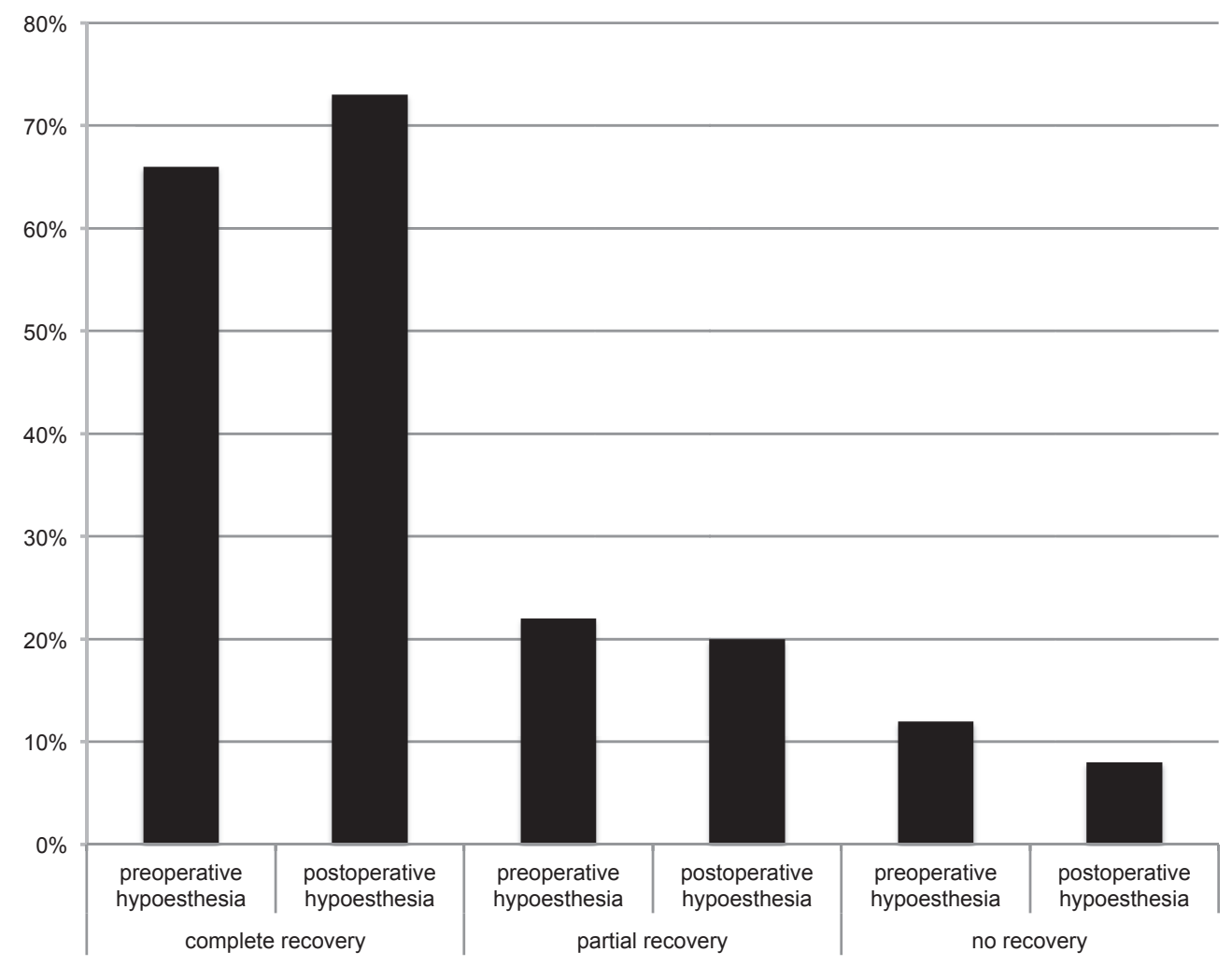

Fig. 3. Demonstrated are the differences in recovery if hypoesthesia was present preoperative or postoperative.

present study supports similar results with worse recovery rates in patients with multiple fractures in the IAN area and especially high rates of hypoesthesia in mandibular angle fractures. A plausible explanation for the higher hypoesthesia rate in mandibular angle fractures is the exposition of the IAN, which is normally protected in the mandibular canal, when fractures occur in the angle area. In these fractures, the fracture line likely runs through the mandibular canal, which leads to straining and compression forces of the IAN. However, in mandibular body and ramus fractures, the IAN is at risk as well, since the IAN is exposed too. Nonetheless the analysis of this study's data did not reveal a significantly higher rate of hypoesthesia in those fractures. The authors suspect that the lack of significance might be explained due to the low number of mandibular body $(\mathrm{n}=25)$ and ramus $(\mathrm{n}=6)$ fractures compared with the high number of angle fractures $(n=163)$. A possible explanation for the low number of mandibular body fractures might be the definition of the anatomical regions for this study. While fractures between the lateral incisor and the second premolar were classified as parasymphyseal, only fractures between the second premolar and the third molar were categorized as body fractures, which leaves a relatively small part of the mandible for the body. In addition there is a high number of angle and a low number of ramus fractures. No distinct border exists between the angle and the ramus. Therefore a certain amount of the fractures of the angle possibly could have been categorized as ramus fractures as well.

Another noteworthy finding is that patients with existent preoperative disturbed nerve continuity showed a significantly lower rate of complete recovery than patients with only postoperative hypoesthesia. This suggests that the initial trauma that directly affects the nerve continuity has a greater impact on the IAN function than the ORIF does.

Furthermore, the recovery rate for body fractures was worse than for fractures that did not affect this part of the mandible. This is a surprising finding, since a study performed by Ruggiero, 1996, states that the mandibular canal might act as guidance for nerve regeneration. A possible explanation for the worse recovery rate might be that the body was defined as the area between the second premolar and the third molar. This area covers a large part of the mandible where the nerve runs in the mandibular canal. The nerve indeed is somewhat protected in the canal, however in mandibular body fractures the fracture line runs through the mandibular canal, which will cause tearing and straining of the nerve. The IAN might also get exposed to sharp bony fragments that can damage or even dissect the IAN. The same mechanism resulted in a higher rate of hypoesthesia in mandibular angle fractures. Nonetheless it has to be stated that only 25 patients with mandibular body fractures were included into the study. Even though statistical significance was achieved, caution has to be executed when analysing such small patient samples.

Limitations of this study include that all data were analysed retrospectively, that IAN function was routinely assessed only by sharp/blunt and two point discrimination and that the follow-up period was limited to 12 months. It has to be noted that retrospective studies can have selection and information bias, which is another limitation of the study.

\section{Conclusion}

Disrupted IAN continuity is seen frequently after mandibular trauma and is a common complication after ORIF of mandibular fractures. Interpersonal altercation was the cause for most of the fractures.

Special attention has to be paid to the IAN when ORIF is performed, since IAN injuries can diminish the quality of life. The present study revealed worse recovery rates of the IAN for older patients as well as for mandibular body fractures. An especially high rate of IAN damage was found for mandibular angle fractures. 
Fortunately mental nerve hypoesthesia has a high potential for recovery. If no recovery occurs after 6 months nerve reconstruction has to be considered.

\section{Conflicts of interest}

None to declare.

\section{Source of funding}

None to declare.

\section{References}

Alpert B, Tiwana PS, Kushner GM: Management of comminuted fractures of the mandible. Oral Maxillofac Surg Clin North Am 21(2): 185-192, 2009

Bede SYH, Ismael WK, Al-Assaf DA, Omer SS: Inferior alveolar nerve injuries associated with mandibular fractures. J Craniofac Surg 23(6): 1776-1778, 2012

Boffano P, Roccia F, Gallesio C, Karagozoglu K, Forouzanfar T: Inferior alveolar nerve injuries associated with mandibular fractures at risk: a two-center retrospective study. Craniomaxillofac Trauma Reconstr 7(4): 280-283, 2014

Boffano P, Roccia F, Zavattero E, Dediol E, Uglešić V, Kovačič Ž et al: European Maxillofacial Trauma (EURMAT) project: a multicentre and prospective study. J Craniomaxillofac Surg 43(1): 62-70, 2015

Chhabra A, Ahlawat S, Belzberg A, Andreseik G: Peripheral nerve injury grading simplified on MR neurography: as referenced to Seddon and Sunderland classifications. Indian J Radiol Imaging 24(3): 217-224, 2014

Chrcanovic BR: Open versus closed reduction: comminuted mandibular fractures. Oral Maxillofac Surg 17(2): 95-104, 2013

Erdmann D, Follmar KE, Debruijn M, Bruno AD, Jung SH, Edelman D, et al: A retrospective analysis of facial fracture etiologies. Ann Plast Surg 60(4): 398-403, 2008

Gassner R, Tuli T, Hachl O, Moreira R, Ulmer H: Craniomaxillofacial trauma in children: a review of 3,385 cases with 6,060 injuries in 10 years. J Oral Maxillofac Surg 62(4): 399-407, 2004
Halpern LR, Kaban LB, Dodson TB: Perioperative neurosensory changes associated with treatment of mandibular fractures. J Oral Maxillofac Surg 62(5): 576-581, 2004

Iatrou I, Theologie-Lygidakis N, Tzerbos F: Surgical protocols and outcome for the treatment of maxillofacial fractures in children: 9 years' experience. J Craniomaxillofac Surg 38(7): 511-516, 2010

Lemke RR, Clark GM, Bays RA, Tiner BD, Rugh JD: Effects of hypesthesia on ora behaviors of the orthognathic surgery patient. J Oral Maxillofac Surg 56(2) 153-157, 1998

Marchena JM, Padwa BL, Kaban LB: Sensory abnormalities associated with mandibular fractures: incidence and natural history. J Oral Maxillofac Surg 56(7): 822-825, 1998

Mayrink G, Moreira RW, Araujo MM: Prospective study of postoperative sensory disturbances after surgical treatment of mandibular fractures. Oral Maxillofac Surg 17(1): 27-31, 2013

Poort LJ, van Neck JW, van der Wal KG: Sensory testing of inferior alveolar nerve injuries: a review of methods used in prospective studies. J Oral Maxillofac Surg 67(2): 292-300, 2009

Renton TF, Wiesenfeld D: Mandibular fracture osteosynthesis: a comparison of three techniques. Br J Oral Maxillofac Surg 34(2): 166-173, 1996

Renzi G, Carboni A, Perugini M, Giovannetti F, Becelli R: Posttraumatic trigemina nerve impairment: a prospective analysis of recovery patterns in a series of 103 consecutive facial fractures. J Oral Maxillofac Surg 62(11): 1341-1346, 2004

Ruggiero SL: Trigeminal nerve injury and repair. N Y State Dent J 62(8): 36-40, 1996

Schultze-Mosgau S, Erbe M, Rudolph D, Ott R, Neukam FW: Prospective study on post-traumatic and postoperative sensory disturbances of the inferior alveolar nerve and infraorbital nerve in mandibular and midfacial fractures. J Craniomaxillofac Surg 27(2): 86-93, 1999

Seddon HJ: A classification of nerve injuries. Br Med J 2(4260): 237-239, 1942

Seemann R, Schicho K, Wutzl A, Koinig G, Poeschl WP, Krennmair G, et al Complication rates in the operative treatment of mandibular angle fractures: 10-year retrospective. J Oral Maxillofac Surg 68(3): 647-650, 2010

Smith JG, Elias LA, Yilmaz Z, Barker S, Shah K, Shah S, et al: The psychosocial and affective burden of posttraumatic neuropathy following injuries to the trigeminal nerve. J Orofac Pain 27(4): 293-303, 2013

Sunderland S: A classification of peripheral nerve injuries producing loss of function. Brain 74(4): 491-516, 195 